\title{
A two-phase hyperelastic-viscoplastic constitutive model for semi-crystalline polymers: Application to polyethylene materials with a variable range of crystal fractions
}

\author{
H. Abdul-Hameed ${ }^{\mathrm{a}, *}$, T. Messager ${ }^{\mathrm{a}}$, G. Ayoub ${ }^{\mathrm{b}}$, F. Zaïri ${ }^{\mathrm{a},{ }^{*}, 1}$, M. Naït-Abdelaziz ${ }^{\mathrm{a}}$, F. Qu ${ }^{\mathrm{a}}$, Zaïri $^{\mathrm{c},{ }^{*}}$ \\ ${ }^{a}$ Université Lille 1 Sciences et Technologies, Laboratoire de Mécanique de Lille (LML), UMR CNRS 8107, F-59650, Villeneuve d'Ascq, France \\ ${ }^{\mathrm{b}}$ Mechanical Engineering Program, Texas A\&M University at Qatar, Doha, Qatar \\ ${ }^{c}$ Pôle de neurochirurgie, Hôpital Universitaire de Lille, rue Emile-Laine, F-59037 Lille, France
}

\begin{abstract}
Polyethylene-based polymers as biomedical materials can contribute to a wide range of biomechanical applications. Therefore, it is important to identify, analyse, and predict with precision their mechanical behaviour. Polyethylene materials are semi-crystalline systems consisting of both amorphous and crystalline phases interacting in a rather complex manner. When the amorphous phase is in the rubbery state, the mechanical behaviour is strongly dependent on the crystal fraction, therefore leading to essentially thermoplastic or elastomeric responses. In this study, the finite deformation stress-strain response of polyethylene materials is modelled by considering these semi-crystalline polymers as twophase heterogeneous media in order to provide insight into the role of crystalline and amorphous phases on the macro-behaviour and on the material deformation resistances, i.e. intermolecular and network resistances. A hyperelastic-viscoplastic model is developed in contemplation of representing the overall mechanical response of polyethylene materials under large deformation. An evolutionary optimization procedure based on a genetic algorithm is developed to identify the model parameters at different strain rates. The identification results show good agreement with experimental data, demonstrating the usefulness of the proposed approach: the constitutive model, with only one set of identified parameters, allows reproducing the stress-strain behaviour of polyethylene materials exhibiting a wide range of crystallinities, the crystal content becoming the only variable of the model.
\end{abstract}

\footnotetext{
*Corresponding author.

E-mail addresses: hemin.abdulhameed@polytech-lille.fr (H. Abdul-Hameed), fahmi.zairi@polytech-lille.fr, fahed.zairi@chru-lille.fr (F. Zaïri).

${ }^{1}$ Tel.: +33 328767460; fax: +33 328767301.
} 


\section{Introduction}

Polyethylene materials have a wide range of applications in orthopaedics due to their mechanical properties as well as their biocompatibility (Ramakrishna et al., 2001; Mano et al., 2004; Atkins et al., 2009; Albano et al., 2011; Fouad and Elleithy, 2011). Polyethylene-based systems (homopolymers, blends and composites), as biomedical materials, are used, for example, in hip and knee joints, as bulk space fillers, and in total disc replacement.

The mechanical behaviour of polyethylene materials is strongly nonlinear and depends on many factors: strain rate (Ayoub et al., 2010), temperature (Aour et al., 2008), loading mode (Hachour et al., 2014), cross-linking, entanglement and, as semi-crystalline systems, on: crystal fraction, crystalline lamellae size and lamellae distribution (Mano et al., 2004; Simis et al., 2006; Seguela, 2007). It is widely known that the true stress-strain curves provide useful information about the deformation mechanisms and yielding behaviour of polymers. Thus, it is important to identify, analyse, and predict with precision their mechanical behaviour (Bergström et al., 2002; Zaïri et al., 2005, 2011; Ponçot et al., 2013).

As a result, the constitutive modelling of semi-crystalline polymers has been widely investigated over the decades. The constitutive models relied on two main approaches. The first approach is based on phenomenological considerations in which the constitutive models are defined by purely mathematical functions, to take into account external factors such as strain rate and temperature, without linking to deformation mechanisms (G'Sell et al., 1999; Zaïri et al., 2006; Ben Hadj Hamouda et al., 2007; Drozdov, 2009; Aour et al., 2008; Dusunceli and Colak, 2008; Regrain et al., 2009; Uchida and Tada, 2011). The alternative approach is physically based; it consists of introducing features of the microstructure in the mathematical development of the constitutive model; to name a few (Nikolov et al., 2002; Ahzi et al., 2003; Ayoub et al., 2011; Hachour et al., 2014). A more exhaustive list can be found in (Abdul-Hameed et al., 2014). Nikolov et al. (2002) and Hachour et al. (2014) presented micromechanical models to predict the small deformation behaviour of high density polyethylene. Ahzi et al. (2003) extended the constitutive model initially developed by Boyce et al. (2000) in order to capture the finite deformation stress-strain behaviour of polyethylene terephthalate above the glass transition temperature by taking into account strain-induced crystallisation. Later, Ayoub et al. (2011) further modified the constitutive model in order to capture the finite deformation stress-strain response of polyethylene materials with different crystal rates. However, the deterministic identification procedure proposed by the authors did not allow optimizing the number of model parameters.

In this contribution, a two-phase constitutive model is proposed to describe the crystal rate influence on the mechanical response of semi-crystalline polymers. We propose to identify the corresponding model parameters by means of a numerical identification procedure based on a genetic algorithm. In this way, we try to reduce the number of model parameters. The applications are achieved by examining an experimental database obtained for semicrystalline polyethylene materials, stretched at different strain rates and above the glass transition temperature, with three crystallinity degrees: a high density polyethylene (HDPE), a low density polyethylene (LDPE) and an ultra-low density polyethylene (ULDPE).

HDPE is a linear (non-branching) semi-crystalline polymer which can be described as having two phases: crystalline and amorphous phases. The crystalline phase is one in which chains fold and orient themselves into highly ordered thick lamellae (Fig. 1a). The HDPE is used as an additive material to ultra-high molecular weight polyethylene to improve its creep resistance and processability (Ramakrishna et al., 2001; Mano et al., 2004; Fouad and Elleithy, 2011). LDPE is a nonlinear (branching) semi-crystalline polymer in which the concentration of branches hinders the crystallisation process, and the crystalline lamellae are less ordered (Fig. 1b), thinner and shorter than in HDPE. LDPE is proposed for the manufacturing of catheters (tubes) due to its flexibility, ease of fabrication while ensuring blood compatibility, non-thrombogenicity and inhibition of infection (Ramakrishna et al., 2001). ULDPE is a two-phase material with non-crystalline predominance (Fig. 1c) and a high level of disordered shortchain branches; the concentrations of short-chain branches are much higher than in LDPE. It restrains the crystallisation process very effectively, resulting in a material with very low density. Due to its mechanical property and biocompatibility, ULDPE is used for food and pharmaceutical packaging films.

The stress-strain response of any semi-crystalline polymer can be related to the microstructure evolution involved in plastic deformation. Four main stages take place during the deformation of semi-crystalline polymers (Schultz, 1984): (i) first, there is a stretching of chains in the amorphous phase, (ii) followed by rotation and (iii) plastic deformation of the lamellae, and (iv) fragmentation of the crystallites leading to the formation of a fibrillar microstructure and to the significant strain hardening observed in the stress-strain response. The resistance to deformation in the semicrystalline polymer can be therefore seen as the sum of an intermolecular resistance related to the first three stages (providing the initial stiffness and the viscoplastic yield) and a microstructure alignment resistance related to the last step. Both resistances are thus the result of a coupling between crystalline and amorphous resistances.

\section{Hyperelastic-viscoplastic constitutive model for semi-crystalline polymers}

Considering semi-crystalline polymers as heterogeneous media, a two-phase constitutive model is developed in this section. The Ayoub et al. (2011) constitutive model has been recently revised by Abdul-Hameed et al. (2014) in the main aim to reduce the number of model parameters. The constitutive model considers basically the stress-strain behaviour as the result of resistances acting in parallel: (A) two (amorphous/crystalline) intermolecular resistances and (B) one alignment/fragmentation resistance. Considering that plastic flow as well as microstructure alignment in semicrystalline polymers can be attributed to overcoming 

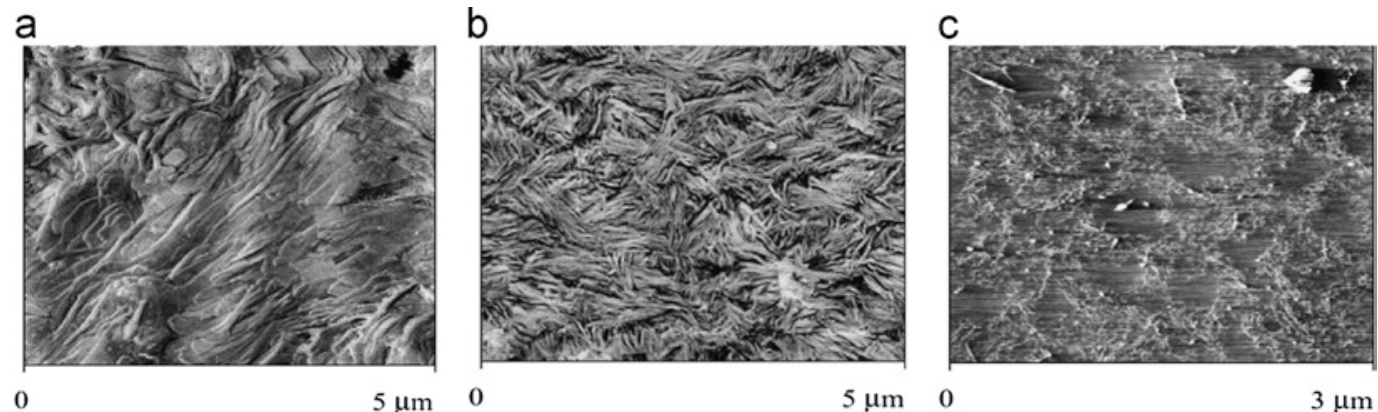

Fig. 1 - Atomic force microscopy images: (a) HDPE, (b) LDPE, and (c) ULDPE. Taken from (Ayoub et al., 2011).

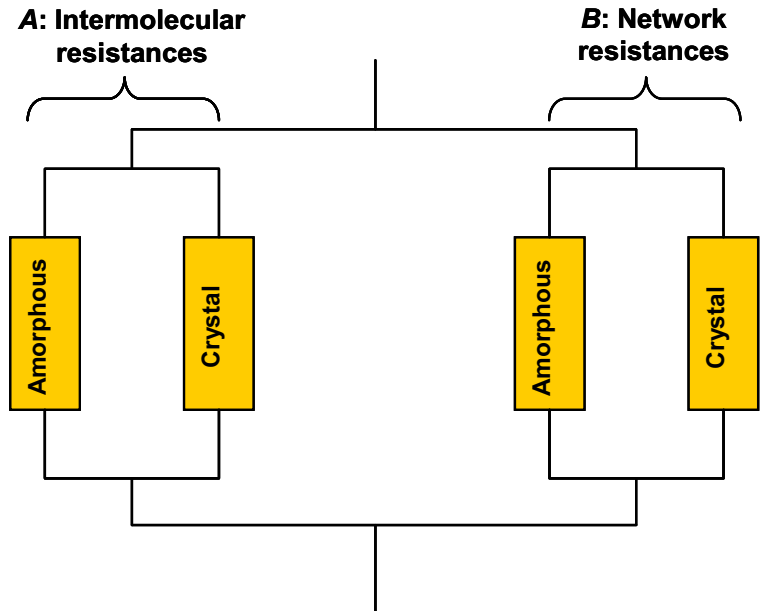

Fig. 2 - Rheological representation of the two-phase model.

resistances in both the amorphous and crystalline phases, we propose in this contribution a further extension of the constitutive model. As shown in Fig. 2, a coupling between crystalline and amorphous phases is explicitly considered in both resistances, which makes a valuable difference with our previous works (Ayoub et al., 2011; Abdul-Hameed et al., 2014).

The intermolecular resistances are represented by linear springs in series with nonlinear dashpots. The linear springs depict the initial elastic response of amorphous and crystalline phases whereas the viscoplastic behaviour is described by the viscous elements. The amorphous network resistance is represented by a Langevin spring in series with a nonlinear dashpot. The Langevin spring corresponds to the amorphous network resistance whereas the viscous element reproduces the amorphous relaxation processes. The crystalline phase contribution in the microstructure alignment resistance is introduced by using a high stiff spring.

\subsection{Kinematics}

As shown by the rheological representation given in Fig. 2, the intermolecular deformation gradient $\mathbf{F}_{\mathrm{A}}$ and the network deformation gradient $\mathbf{F}_{B}$ are equal to the total deformation gradient $\mathbf{F}$. The corresponding crystal and amorphous deformation gradients are also identical for each branch:

$\mathbf{F}_{\mathrm{A}}=\mathbf{F}_{\mathrm{Ac}}=\mathbf{F}_{\mathrm{A} a}$ and $\quad \mathbf{F}_{\mathrm{B}}=\mathbf{F}_{\mathrm{BC}}=\mathbf{F}_{\mathrm{B} a}$ where the subscripts (c) and (a) denote the crystalline and amorphous phases, respectively. The deformation gradient tensors can be decomposed multiplicatively into elastic (network) and plastic (flow) deformation gradients for both resistances $\mathrm{A}$ and $\mathrm{B}$ :

$\mathbf{F}_{\mathrm{Ac}}=\mathbf{F}_{\mathrm{Ac}}^{e} \mathbf{F}_{\mathrm{Ac}}^{p}$ and $\mathbf{F}_{\mathrm{Aa}}=\mathbf{F}_{\mathrm{A} a}^{e} \mathbf{F}_{\mathrm{Aa}}^{p}$

$\mathbf{F}_{\mathrm{BC}}=\mathbf{F}_{\mathrm{BC}}^{\mathrm{N}} \mathbf{F}_{\mathrm{BC}}^{\mathrm{F}} \quad$ and $\quad \mathbf{F}_{\mathrm{Ba}}=\mathbf{F}_{\mathrm{Ba}}^{\mathrm{N}} \mathbf{F}_{\mathrm{B} a}^{\mathrm{F}}$

According to the polar decomposition theorem, both elastic $\mathbf{F}_{\mathrm{A}}^{e}$ and plastic $\mathbf{F}_{\mathrm{A}}^{p}$ deformation gradients of resistance A can be further decomposed into stretch and rotation movements:

$\mathbf{F}_{\mathrm{Ac}}^{e}=\mathbf{V}_{\mathrm{Ac}}^{e} \mathbf{R}_{\mathrm{Ac}}^{e}$ and $\mathbf{F}_{\mathrm{Aa}}^{e}=\mathbf{V}_{\mathrm{Aa}}^{e} \mathbf{R}_{\mathrm{Aa}}^{e}$

$\mathbf{F}_{\mathrm{Ac}}^{p}=\mathbf{V}_{\mathrm{Ac}}^{p} \mathbf{R}_{\mathrm{Ac}}^{p}$ and $\mathbf{F}_{\mathrm{Aa}}^{p}=\mathbf{V}_{\mathrm{Aa}}^{p} \mathbf{R}_{\mathrm{Aa}}^{p}$

In the same manner, both network $\mathbf{F}_{B}^{N}$ and flow $\mathbf{F}_{B}^{F}$ deformation gradients of resistance $B$ can be decomposed into stretch and rotation movements:

$\mathbf{F}_{\mathrm{Ba}}^{\mathrm{N}}=\mathbf{V}_{\mathrm{Ba}}^{\mathrm{N}} \mathbf{R}_{\mathrm{Ba}}^{\mathrm{N}}$

$\mathbf{F}_{\mathrm{Ba}}^{\mathrm{F}}=\mathbf{V}_{\mathrm{Ba}}^{\mathrm{F}} \mathbf{R}_{\mathrm{B} a}^{F}$

Because only a spring acts in the crystalline branch of the resistance $B$, the flow deformation gradient in the crystalline phase is equal to unity, i.e. $\mathbf{F}_{B C}^{F}=\mathbf{I}$. The velocity gradient tensor of resistance $A$ is $\mathbf{L}_{A c}=\dot{\mathbf{F}}_{A C} \mathbf{F}_{A c}^{-1}$ for the crystalline phase and $\mathbf{L}_{A a}=\dot{\mathbf{F}}_{A a} \mathbf{F}_{A a}^{-1}$ for the amorphous phase. They can be decomposed into elastic and plastic parts:

$\mathbf{L}_{A c}=\mathbf{L}_{A c}^{e}+\mathbf{L}_{A c}^{p}=\dot{\mathbf{F}}_{A c}^{e} \mathbf{F}_{A c}^{e-1}+\mathbf{F}_{A c}^{e} \dot{\mathbf{F}}_{\mathrm{Ac}}^{p} \mathbf{F}_{\mathrm{Ac}}^{p-1} \mathbf{F}_{\mathrm{Ac}}^{e-1}$

$\mathbf{L}_{\mathrm{Aa}}=\mathbf{L}_{\mathrm{Aa}}^{e}+\mathbf{L}_{\mathrm{A} a}^{p}=\dot{\mathbf{F}}_{\mathrm{Aa}}^{e} \mathbf{F}_{\mathrm{Aa}}^{e-1}+\mathbf{F}_{\mathrm{Aa}}^{e} \dot{\mathbf{F}}_{\mathrm{Aa}}^{p} \mathbf{F}_{\mathrm{Aa}}^{p-1} \mathbf{F}_{\mathrm{Aa}}^{e-1}$

$\mathbf{L}_{\mathrm{Ac}}^{p}=\mathbf{D}_{\mathrm{Ac}}^{p}+\mathbf{W}_{\mathrm{Ac}}^{p}$ and $\quad \mathbf{L}_{\mathrm{Aa}}^{p}=\mathbf{D}_{\mathrm{Aa}}^{p}+\mathbf{W}_{\mathrm{Aa}}^{p}$

where $\mathbf{D}_{\mathrm{Ac}}^{p}$ and $\mathbf{D}_{\mathrm{Aa}}^{p}$ are the plastic deformation rates, and $\mathbf{W}_{\mathrm{Ac}}^{p}$ and $\mathbf{w}_{\mathrm{Aa}}^{p}$ are the plastic spins. The inelastic flow is assumed to be irrotational and the associated spins are taken equal to zero $\left(\mathbf{W}_{\mathrm{Ac}}^{p}=\mathbf{W}_{\mathrm{Aa}}^{p}=0\right)$. The inelastic flow is assumed to be incompressible, i.e. $\operatorname{det} \mathbf{F}_{\mathrm{Ac}}^{p}=\operatorname{det} \mathbf{F}_{\mathrm{Aa}}^{p}=1$.

The velocity gradient tensor of resistance B is $\mathbf{L}_{\mathrm{Ba}}=\dot{\mathbf{F}}_{\mathrm{Ba}} \mathbf{F}_{\mathrm{Ba}}^{-1}$ and can be decomposed into network and flow parts:

$\mathbf{L}_{\mathrm{Ba}}=\mathbf{L}_{\mathrm{Ba}}^{\mathrm{N}}+\mathbf{L}_{\mathrm{Ba}}^{\mathrm{F}}=\dot{\mathbf{F}}_{\mathrm{Ba}}^{\mathrm{N}} \mathbf{F}_{\mathrm{Ba}}^{\mathrm{N}-1}+\mathbf{F}_{\mathrm{B} a}^{\mathrm{N}} \dot{\mathbf{F}}_{\mathrm{B} a}^{\mathrm{F}} \mathbf{F}_{\mathrm{B} a}^{\mathrm{F}-1} \mathbf{F}_{\mathrm{Ba}}^{\mathrm{N}-1}$

$\mathbf{L}_{\mathrm{Ba}}^{F}=\mathbf{D}_{\mathrm{Ba}}^{F}+\mathbf{W}_{\mathrm{B} a}^{F}$ 
where $\mathbf{D}_{\mathrm{Ba}}^{\mathrm{F}}$ is the molecular relaxation rate and $\mathbf{W}_{\mathrm{Ba}}^{\mathrm{F}}$ is the flow spin. Again, without loss of generality, the inelastic spin in the amorphous phase is assumed to be null $\left(\mathbf{W}_{B a}^{F}=0\right)$. The inelastic flow in the amorphous phase is assumed to be incompressible, i.e. $\operatorname{det} \mathbf{F}_{\mathrm{Ba}}^{\mathrm{F}}=1$.

The Cauchy stress tensor is deducted from the summation of the intermolecular Cauchy stress tensor $\mathbf{T}_{A}$ and the network Cauchy stress tensor $\mathbf{T}_{B}$ :

$\mathbf{T}=\mathbf{T}_{\mathrm{A}}+\mathbf{T}_{\mathrm{B}}$

\subsection{Model formulation}

\subsubsection{Resistance A}

The structure of resistance $A$ is divided into crystalline and amorphous branches. The crystalline and amorphous Cauchy stresses are expressed as:

$\mathbf{T}_{\mathrm{Ac}}=J_{\mathrm{Ac}}^{-1} \mathbf{C}_{\mathrm{c}}^{e} \ln \left(\mathbf{V}_{\mathrm{Ac}}^{e}\right)$

$\mathbf{T}_{\mathrm{A} a}=\mathrm{J}_{\mathrm{A} a}^{-1} \mathbf{C}_{a}^{e} \ln \left(\mathbf{V}_{\mathrm{A} a}^{e}\right)$

where $J_{\mathrm{Ac}}$ and $J_{\mathrm{Aa}}$ are the elastic volume changes, $\mathbf{C}_{\mathrm{c}}^{e}$ and $\mathbf{C}_{a}^{e}$ are the isotropic elasticity tensors (depending on the elastic moduli $E_{c}$ and $E_{a}$, and on the Poisson's ratios $v_{c}$ and $v_{a}$ ), and In $\left(\mathbf{V}_{A c}^{e}\right)$ and In $\left(\mathbf{V}_{\mathrm{Aa}}^{e}\right)$ are the Hencky strains. The plastic strain rate tensors for the crystalline and amorphous phases are:

$\mathbf{D}_{A c}^{P}=\dot{\gamma}_{A c}^{p} \mathbf{T}_{A c}^{\prime} / \sqrt{2} \tau_{A c}$

$\mathbf{D}_{\mathrm{Aa}}^{P}=\dot{\gamma}_{\mathrm{Aa}}^{p} \mathbf{T}_{\mathrm{Aa}}^{\prime} / \sqrt{2} \tau_{\mathrm{Aa}}$

in which $\mathbf{T}_{\mathrm{Ac}}^{\prime}$ and $\mathbf{T}_{\mathrm{Aa}}^{\prime}$ are the deviatoric parts of the Cauchy stress tensors $\mathbf{T}_{A c}$ and $\mathbf{T}_{\mathrm{Aa}}, \tau_{\mathrm{Ac}}$ and $\tau_{\mathrm{Aa}}$ are the effective stresses, and $\dot{\gamma}_{\mathrm{Ac}}^{p}$ and $\dot{\gamma}_{\mathrm{Aa}}^{p}$ are the plastic shear strain rates written as:

$\dot{\gamma}_{\mathrm{Ac}}^{\mathrm{P}}=\dot{\gamma}_{\mathrm{OA}}^{\mathrm{c}} \exp \left[-\Delta G_{\mathrm{c}}\left(1-\tau_{\mathrm{Ac}} / \mathrm{s}_{\mathrm{c}}\right) / k \theta\right]$

$\dot{\gamma}_{\mathrm{Aa}}^{\mathrm{P}}=\dot{\gamma}_{\mathrm{OA}}^{a} \exp \left[-\Delta G_{a}\left(1-\tau_{\mathrm{Aa}} / \mathrm{s}_{a}\right) / k \theta\right]$

where $\dot{\gamma}_{o \mathrm{~A}}^{\mathrm{c}}$ and $\dot{\gamma}_{\mathrm{oA}}^{a}$ are pre-exponential factors, $\Delta G_{c}$ and $\Delta G_{a}$ are the activation energies, $s_{c}$ and $s_{a}$ are the shear resistances, $k$ is the Boltzmann constant and $\theta$ is the absolute temperature. Both activation energies and shear resistances capture barriers to deformation in the respective phases: $\Delta G_{c}$ and $s_{c}$ capture barrier to crystallographic shear in the crystalline phase, and $\Delta G_{a}$ and $s_{a}$ capture barrier to molecular chain segment rotation in the amorphous phase. The effects of both phases are summed to determine the intermolecular Cauchy stress $\mathbf{T}_{\mathrm{A}}$ :

$\mathbf{T}_{\mathrm{A}}=\left(\chi_{\mathrm{cv}}\right)^{\beta_{1}} \mathbf{T}_{\mathrm{Ac}}+\left(1-\chi_{\mathrm{cv}}\right)^{\beta_{1}} \mathbf{T}_{\mathrm{Aa}}$

where $\chi_{c v}$ is the crystal volume fraction, and $\beta_{I}$ is introduced to take into account the intermolecular mechanical interactions between the two phases.

\subsubsection{Resistance $B$}

The strain hardening response may be attributed to both molecular and crystallographic orientations; thus we propose to treat the effect of crystalline and amorphous phases separately. (1) The amorphous phase in the resistance $B$. The stress-strain relationship in the amorphous phase during the strain hardening stage can be described using a limiting chain extensibility model (Arruda and Boyce, 1993):

$\mathbf{T}_{\mathrm{B} a}=\mathrm{J}_{\mathrm{B} a}^{-1} C_{a} / 3 \sqrt{\mathrm{N}} / \lambda_{\mathrm{N} a} \ell^{-1}\left(\lambda_{\mathrm{N} a} / \sqrt{\mathrm{N}}\right)\left[\mathbf{B}_{a}^{\mathrm{N}}-\lambda_{\mathrm{N} a}^{2} \mathbf{I}\right]$

in which $J_{B a}$ is the amorphous network volume change, $C_{a}$ is the rubbery modulus of the amorphous phase, $\sqrt{N}$ is the limiting chain extensibility parameter ( $N$ being the number of amorphous molecular units between entanglements and cross-links), $\ell^{-1}$ is the inverse Langevin function given by $\ell(x)=\operatorname{coth}(x)-1 / x, \mathbf{B}_{a}^{N}$ is the amorphous network left Cauchy-Green tensor $\left(\mathbf{B}_{a}^{N}=\mathbf{F}_{B a}^{N} \mathbf{F}_{B a}^{N-T}\right)$, and $\lambda_{N a}$ is given by:

$\lambda_{\mathrm{Na}}=\left[\operatorname{trace}\left(\mathbf{F}_{\mathrm{Ba}}^{\mathrm{N}} \mathbf{F}_{\mathrm{Ba}}^{\mathrm{N}-\mathrm{T}}\right) / 3\right]^{1 / 2}$

The molecular relaxation rate is written as:

$\mathbf{D}_{\mathrm{Ba}}^{\mathrm{F}}=\dot{\gamma}_{\mathrm{Ba}}^{\mathrm{F}} \mathbf{T}_{\mathrm{Ba}}^{\prime} / \sqrt{2} \tau_{\mathrm{Ba}}$

where $\mathbf{T}_{B a}^{\prime}$ is the deviatoric part of the Cauchy stress tensor $\mathbf{T}_{\mathrm{Ba}}, \tau_{\mathrm{Ba}}$ represents the effective stress, and $\dot{\gamma}_{\mathrm{Ba}}^{\mathrm{F}}$ is the rate of relaxation:

$\dot{\gamma}_{\mathrm{Ba}}^{\mathrm{F}}=\mathrm{C}\left(1 /\left(\lambda_{\mathrm{Ba}}^{\mathrm{F}}-1\right)\right) \tau_{\mathrm{Ba}}$

where $C$ is a temperature-dependent relaxation parameter. The term $\lambda_{\mathrm{Ba}}^{\mathrm{F}}$ is given by:

$\lambda_{\mathrm{Ba}}^{\mathrm{F}}=\left[\operatorname{trace}\left(\mathbf{F}_{\mathrm{Ba}}^{\mathrm{F}} \mathbf{F}_{\mathrm{Ba}}^{F_{-} \mathrm{T}}\right) / 3\right]^{1 / 2}$

(2) The crystalline phase in the resistance B. During the strain hardening stage, the crystalline domains involve several elementary microstructural deformation mechanisms, which are complex to model. Considering the crystalline phase stiffness as a first-order factor in the microstructure alignment resistance, a simple Neo-Hookean formulation is used to represent the Cauchy stress in the crystalline phase:

$\mathbf{T}_{B C}=J_{B C}^{-1} 2 C_{C} \mathbf{B}_{C}$

in which $J_{B C}$ is the crystal network volume change, $C_{C}$ is a constant and $\mathbf{B}_{C}$ is the crystal left Cauchy-Green tensor: $\mathbf{B}_{c}=\mathbf{F}_{B C} \mathbf{F}_{B C}^{T}$. The fragmentation process having an effect on the crystalline domain perfection, the crystal stiffness is expected to be degraded and the $C_{c}$ value to be lower than the crystal elastic modulus value in the intermolecular resistance.

The effects of both phases are summed to determine the network Cauchy stress $\mathbf{T}_{B}$ :

$\mathbf{T}_{\mathrm{B}}=\left(\chi_{\mathrm{cv}}\right)^{\beta_{\mathrm{N}}} \mathbf{T}_{\mathrm{BC}}+\left(1-\chi_{\mathrm{cv}}\right)^{\beta_{\mathrm{N}}} \mathbf{T}_{\mathrm{B} a}$

where $\chi_{c v}$ is again the crystal volume fraction and $\beta_{N}$ is introduced to take into account the network mechanical interactions between the two phases.

\section{Numerical identification of parameters}

This section details the curve fitting problem computation. The experiment-based identification of model parameters can be expressed as an optimization search: minimizing the discrepancies between the experimental and numerical stress-strain results. This class of optimization problems, of mixed variables, appears to be non-convex. Its reasonable numerical solving then requires the use of global robust search approaches. 


\subsection{Formulation of the optimization problem}

The curve fitting problem under consideration tends to minimize the gaps between experimental and numerical stress-strain curves. In other words, the objective consists of finding the best set and matching the experimental material behaviour of numerical model parameters of the following form:

$X=\left\{E_{a}, E_{c}, \Delta G_{a}, \Delta G_{c}, s_{a}, s_{c}, C, N, C_{a}, C_{c}\right\}$

Considering the experimental database available for a particular tested material, this curve fitting task has to be performed for $N_{V}$ stress-strain curves, $N_{V}$ being the number of applied strain rates. Moreover, each curve induces $N_{P, L}$ experimental points (noticed $T_{L, K}^{E x p}$ ) for the Lth velocity, with $1 \leq L \leq N_{V}$ and $1 \leq K \leq N_{P, L}$. The corresponding numerical stress values are then denoted by $T_{L, K}^{N u m}=T_{L, K}^{N u m}(X)$. By this means, the formulation of the optimization problem can be expressed as follows: find the best parameter set X, minimizing the discrepancies between the experimental $T_{L, K}^{E x p}$ and numerical $T_{L, K}^{N u m}=T_{L, K}^{N u m}(X)$ points, these discrepancies being evaluated using the following objective function:

$\left.f(X)=\sum_{L=1}^{N_{V}} \sum_{K=1}^{N_{P, L}}\left\|T_{L, K}^{N u m}-T_{L, K}^{E x p}\right\| / N_{P, L}\right) / N_{V}$

This reasonable objective function $f$, being normalised by the terms $1 / N_{V}$ and $1 / N_{P, L}$, is then not sensitive to the numbers of strain rates and to the corresponding numbers of experimental points $T_{L, K}^{E x p}$ under consideration as pointed out by Pyrz and Zaïri (2007).

As underlined in the literature (Pyrz and Zaïri, 2007; Chaparro et al., 2008; Mahmoudi et al., 2011), the computational solving of such an optimization problem involves several difficulties:

- The mechanical behaviours of the studied materials are strongly non-linear.

- The experimental databases considered induce a large number of experimental points.

- The model parameter set $X=\left\{E_{a}, E_{c}, \Delta G_{a}, \Delta G_{c}, s_{a}, S_{c}\right.$, $\left.C, N, C_{a}, C_{c}\right\}$ implies mixed design variables, both real and integers.

By this means, such mixed optimization problems exhibit local optima in non-convex design spaces of large cardinality. The corresponding numerical approach to identify the model parameters therefore requires specific global search procedures. For the present work, a genetic algorithm (GA) strategy, detailed in the next subsection, has been computed.

\subsection{Numerical identification tool}

The GA belongs to the general class of evolutionary algorithms (EA): they are general purpose, stochastic search methods inspired by the natural evolution process (Michalewicz, 1999). They are naturally suited to solve multi-modal search problems of high cardinality, exhibiting both real and integer design variables. They are thus widely used to solve a large variety of optimization tasks in engineering sciences; numerous works of

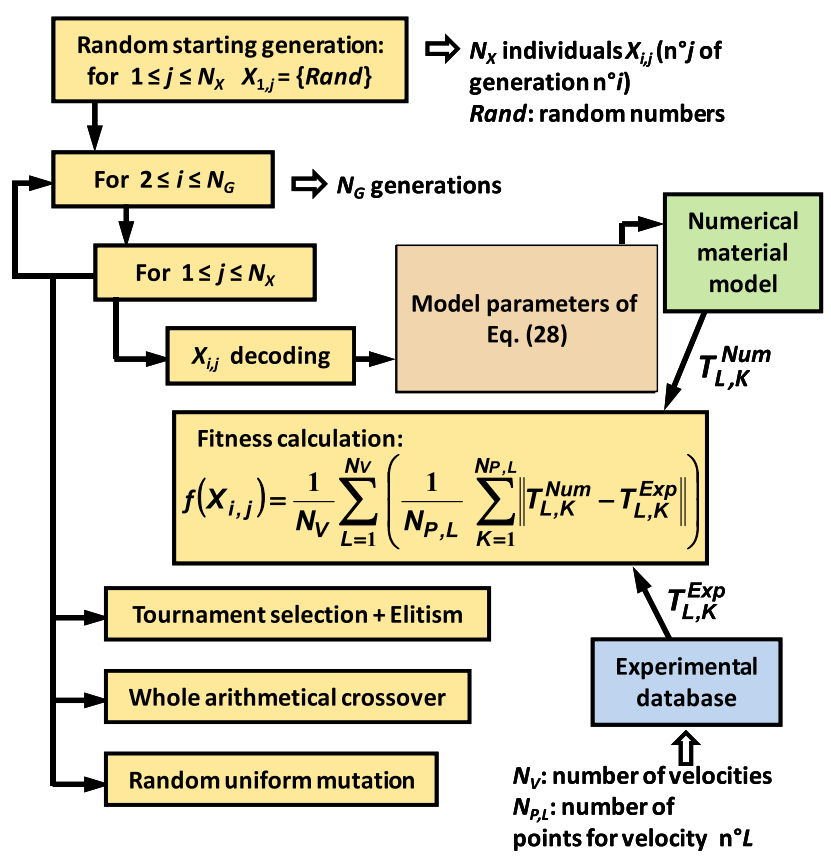

Fig. 3 - Numerical parameter identification flowchart.

the literature have demonstrated the usefulness and efficiency of such procedures (Miettinen et al., 1999; Gen and Cheng, 2000).

As can be reminded, the main idea of this class of search methods consists in processing at a time a fixed number of potential solutions called population of individual $X$. The performance of each of these potential solutions $X$ is characterized by its fitness value. For the present work, this fitness has been chosen to be equal to $1 / f$, see. Eq. (29). According to evolutionary theories, the fittest individuals (i.e. leading to the greatest fitness) are likely to be selected to be "parents", to engender a new generation of solutions by recombining their features. This simulated process of evolution operates using a set of biologically inspired stochastic operators. First, the crossover procedure recombines the genetic characteristics (i.e. the optimization parameters) of some selected parents, thus producing "children" individuals expected to improve the optimization search. Some of the children are next arbitrarily changed by the mutation operator, avoiding a population focused on a local optimum. This simulated reproduction is renewed for a fixed number of generations. At last, the GA yields the best individual found during the evolution process.

Due to the mixed nature of the design variables of the present identification problem, the GA developed for this study is canonical, directly manipulating the design parameters $X$, without any binary encoding, as recommended by the literature (Michalewicz, 1999). Typical corresponding genetic operators have been used: starting from a randomly created population, the selection of parents for mating is based on tournament ranking using random pairs. Moreover, the elitist procedure automatically duplicates the best individual in the new generation. The new solutions are produced by recombining the individuals of the selected individuals using the whole arithmetical crossover (applied with the probability 75\%) and the random mutation (using a probability of $5 \%$ ). The details of these genetic operators can be found in (Michalewicz, 1999; 

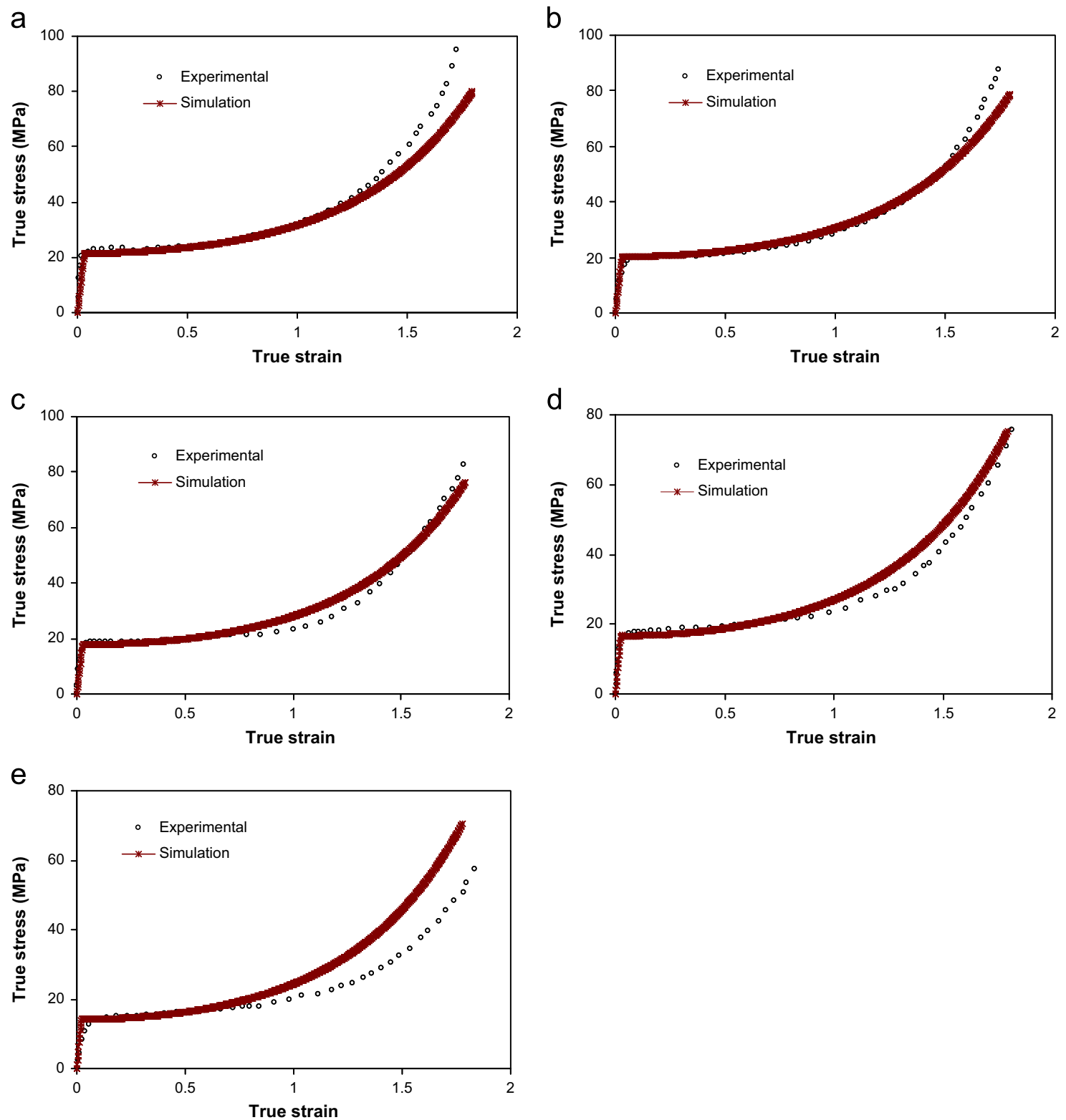

Fig. 4 - Experimental vs simulated stress-strain response of HDPE: (a) at strain rate of $0.01 \mathrm{~s}^{-1}$, (b) at strain rate of $0.005 \mathrm{~s}^{-1}$, (c) at strain rate of $0.001 \mathrm{~s}^{-1}$, (d) at strain rate of $0.0005 \mathrm{~s}^{-1}$, and (e) at strain rate of $0.0001 \mathrm{~s}^{-1}$.

Gen and Cheng, 2000). The corresponding computed numerical identification tool is illustrated in Fig. 3. At last, it should be underlined that, due to the stochastic nature of the GA, each identification problem studied thereafter was analysed using 10 successive runs of optimization.

\section{Experimental data and constitutive model results}

In this section, the two-phase constitutive model parameters are identified to represent the mechanical behaviour of semicrystalline materials stretched under large deformation.

\subsection{Materials and experiments}

Semi-crystalline polyethylene materials with three different grades were considered for the present study: a high density polyethylene (HDPE) with crystal volume fraction $\chi_{\mathrm{cv}}=0.724$, a low density polyethylene (LDPE) with $\chi_{\mathrm{cv}}=0.3$, and an ultralow density polyethylene (ULDPE) with $\chi_{\mathrm{cu}}=0.15$. The monotonic stress-strain response was obtained under uniaxial tension by means of a universal testing machine Instron5800 connected to a video-controlled system able to maintain a constant local true axial strain rate. The tests were achieved at room temperature and for five true axial strain rates: $0.01,0.005,0.001,0.0005$, and $0.0001 \mathrm{~s}^{-1}$. The details on the experimental procedure and results are given elsewhere (Ayoub et al., 2011). 
a
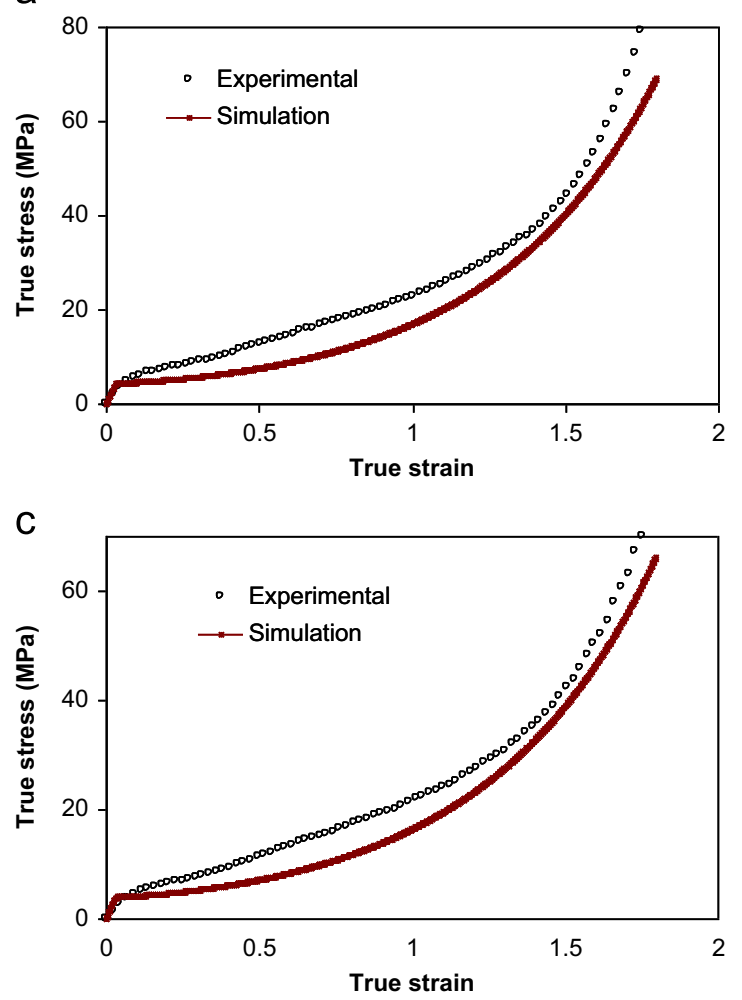

e

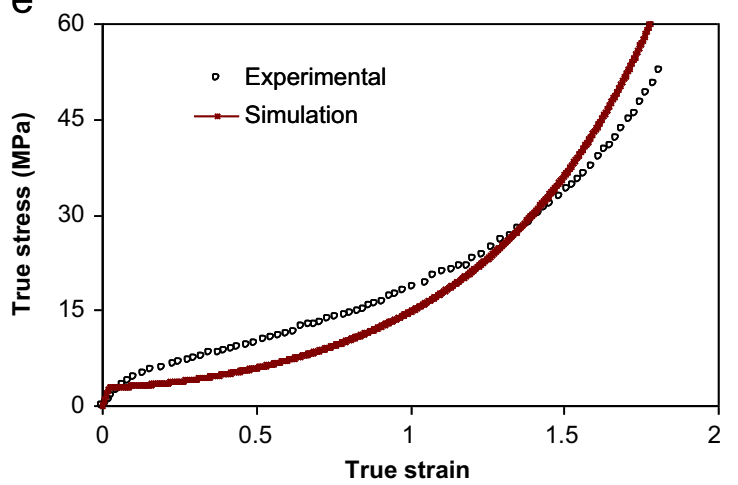

b

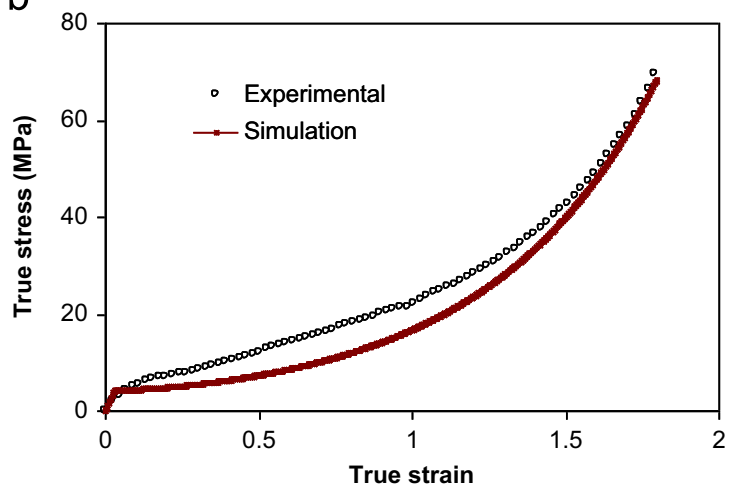

$d$

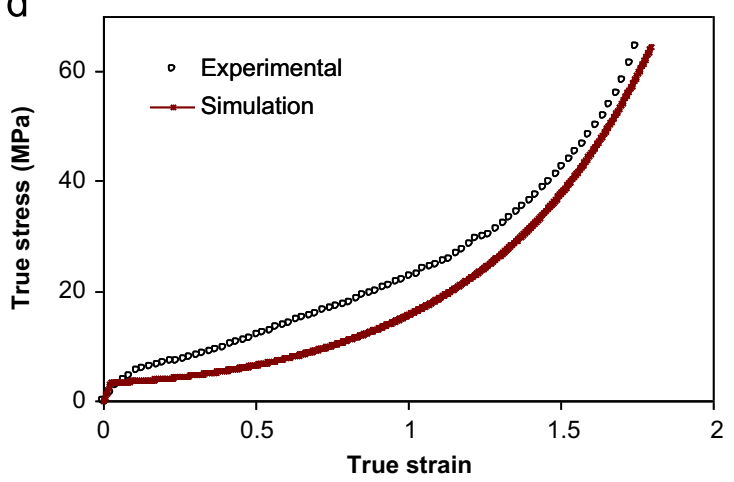

Fig. 5 - Experimental vs simulated stress-strain response of LDPE: (a) at strain rate of $0.01 \mathrm{~s}^{-1}$, (b) at strain rate of $0.005 \mathrm{~s}^{-1}$, (c) at strain rate of $0.001 \mathrm{~s}^{-1}$, (d) at strain rate of $0.0005 \mathrm{~s}^{-1}$, and (e) at strain rate of $0.0001 \mathrm{~s}^{-1}$.

Figs. 4-6 present the experimental stress-strain curves of the three polyethylene grades. As can be seen, a decrease in crystal content leads to a transition from the typical thermoplastic behaviour to the characteristic elastomer behaviour. Such notable behaviour differences enable one to identify next the respective influence of amorphous and crystal rheological parameters.

\subsection{Experimental data vs. constitutive model results}

The sensitivity to the crystallinity effects of the constitutive model proposed in this paper has been evaluated. In that way, the identification of the model parameters has been treated considering the mechanical behaviour dominated either by the crystalline or by the amorphous phase: HDPE and ULDPE materials, respectively. This optimization problem, achieved using the numerical tool illustrated in Fig. 3, is thus based on the ten experimental stress-strain curves depicted in Figs. 4 and 6. The elastic properties of the crystalline and amorphous phases are prescribed; the values are those given in Ayoub et al. (2011) and Abdul-Hameed et al. (2014). In addition, the interaction parameters $\beta_{I}$ and $\beta_{\mathrm{N}}$ have been identified for the HDPE and ULDPE materials using the same numerical optimization tool.

The model parameter values obtained by identification are listed in Table 1, and the corresponding interaction parameters are detailed in Table 2.

The corresponding stress-strain curves deduced from the proposed constitutive model are depicted in Figs. 4 and 6 for the five true axial strain rates. It is interesting to note that the proposed two-phase model is able to accurately reproduce the ULDPE and HDPE mechanical responses using the 

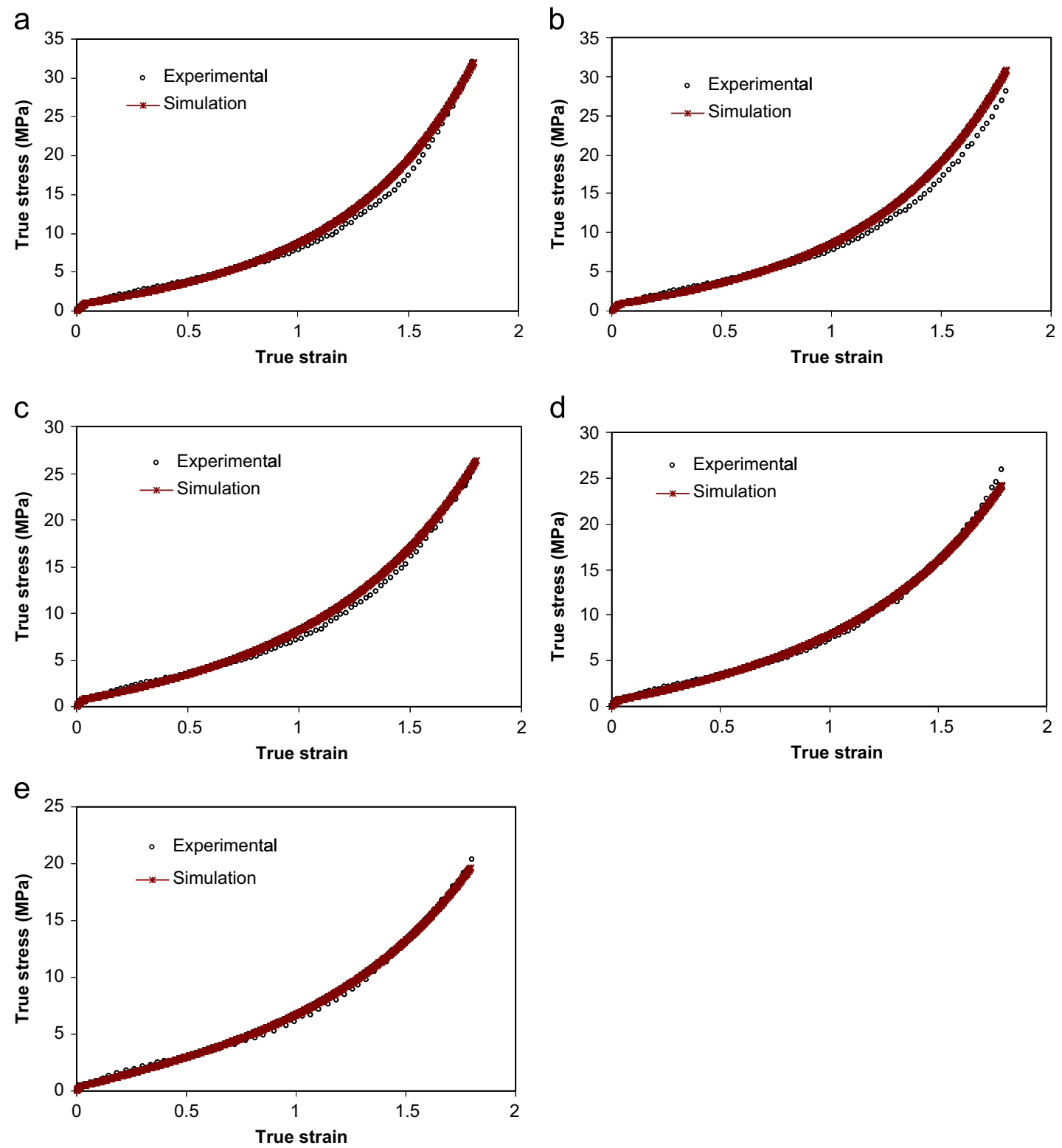

Fig. 6 - Experimental vs simulated stress-strain response of ULDPE: (a) at strain rate of $0.01 \mathrm{~s}^{-1}$, (b) at strain rate of $0.005 \mathrm{~s}^{-1}$, (c) at strain rate of $0.001 \mathrm{~s}^{-1}$, (d) at strain rate of $0.0005 \mathrm{~s}^{-1}$, and (e) at strain rate of $0.0001 \mathrm{~s}^{-1}$.
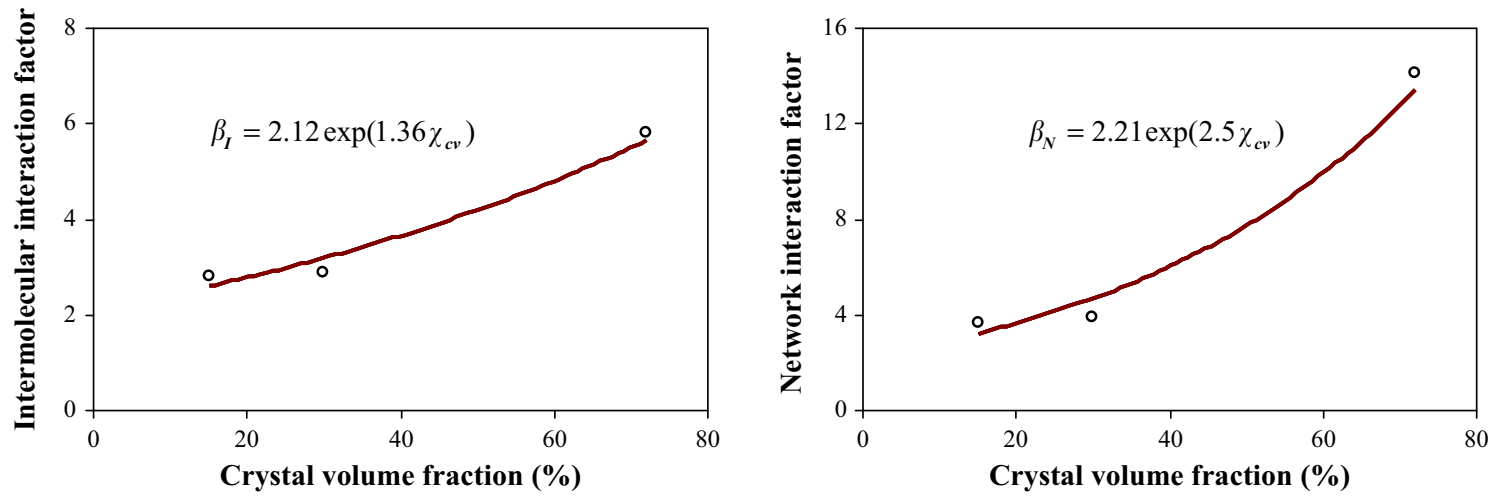

Fig. 7 - Evolution of mechanical interaction parameters according to crystal rate. 
Table 1 - Identified crystalline and amorphous model parameters.

\begin{tabular}{lllll}
$\Delta G_{a}(\mathrm{~J})$ & $\mathrm{s}_{a}(\mathrm{MPa})$ & $\mathrm{C}_{a}(\mathrm{MPa})$ & $\mathrm{C}(\mathrm{MPa} . \mathrm{s})^{-1}$ & $\mathrm{~N}$ \\
\hline $2.62 \times 10^{-19}$ & 6.88 & 1.7 & $1.2996 \times 10^{-7}$ & 202 \\
$\Delta G_{\mathrm{c}}(\mathrm{J})$ & $\mathrm{s}_{\mathrm{c}}(\mathrm{MPa})$ & $\mathrm{C}_{\mathrm{c}}(\mathrm{MPa})$ & & \\
\hline $1.29 \times 10^{-19}$ & 190.73 & 239.13 & & \\
\hline
\end{tabular}

Table 2 - Identified intermolecular $\left(\beta_{\mathrm{I}}\right)$ and network $\left(\beta_{\mathrm{N}}\right)$ mechanical interaction parameters.

\begin{tabular}{crll} 
Parameter & HDPE & LDPE & ULDPE \\
\hline $\boldsymbol{\beta}_{\mathrm{I}}$ & 5.8 & 2.9 & 2.8 \\
$\boldsymbol{\beta}_{\mathrm{N}}$ & 14.1 & 3.9 & 3.7
\end{tabular}

parameter set reported in Table 1 and the interaction parameters detailed in Table 2. As can be seen in Figs. 4 and 6, the numerical and experimental curves are in good agreement. Such results confirm the relevance of both the proposed twophase model and the identification approach.

Next, the optimized parameters depicted in Table 1 have been used as input data in the proposed two-phase model to predict the LDPE stress-strain response. For this material, the interaction parameters were the outcome of a standard fitting procedure based on the minimization of differences between model results and experimental data. The obtained values are given in Table 2. The numerical corresponding stress-strain curves are shown in Fig. 5 in comparison with the experimental data for the five true axial strain rates. As can be seen, the numerical results match the experimental data in a reasonable extent. As shown in Fig. 7, the evolution of both interaction parameters $\beta_{I}$ and $\beta_{N}$ is monotonic. The key assumption of the adopted micromechanical approach is to consider that the crystalline and amorphous domains mechanically behave in the same manner whatever the crystal content; only the interactions between the two phases change. It is found that the mechanical interaction parameters, in the plastic yielding (intermolecular) and molecular stretching/orientation (network) regions, follow an exponential law with the crystal content. The proposed constitutive model can be used to estimate the largestrain mechanical response of any semi-crystalline polyethylene material with a crystal fraction included in the investigated range. In a forthcoming paper, the constitutive model will be used to predict the response of multi-layered polyethylene specimens with different crystal fractions, in the aim to design orthopaedics implants involving graded mechanical properties.

\section{Conclusion}

In this investigation, a two-phase hyperelastic-viscoplastic constitutive model developed to simulate the finite deformation mechanical response of semi-crystalline polymers is proposed. This model is basically decomposed into intermolecular resistances and microstructure alignment resistances, by considering explicitly the contribution of the crystalline phase. The model parameters have been deduced using a numerical identification tool. This approach has been applied to the study of three grades of polyethylene exhibiting a wide variety of crystal rates. The results obtained have demonstrated the significance and the accuracy of the developed two-phase model and the identification procedure. It could be underlined that a reduced set of model parameters enables the simulation of the totality of the studied polyethylene grades.

\section{R E F E R E N C E S}

Abdul-Hameed, H., Messager, T., Zaïri, F., Naït-Abdelaziz, M., 2014. Large-strain viscoelastic-viscoplastic constitutive modeling of semi-crystalline polymers and model identification by deterministic/evolutionary approach. Comput. Mater. Sci. 90, 241-252.

Ahzi, S., Makradi, A., Gregory, R.V., Edie, D.D., 2003. Modeling of deformation behavior and strain-induced crystallization in poly(ethylene terephthalate) above the glass transition temperature. Mech. Mater. 35, 1139-1148.

Albano, C., Perera, R., Cataño, L., Karam, A., González, G., 2011. Prediction of mechanical properties of composites of HDPE/ HA/EAA. J. Mech. Behav. Biomater. 4, 467-475.

Aour, B., Zaïri, F., Naït-Abdelaziz, M., Gloaguen, J.M., Rahmani, O., Lefebvre, J.M., 2008. A computational study of die geometry and processing conditions effects on equal channel angular extrusion of a polymer. Inter. J. Mech. Sci. 50, 589-602.

Arruda, E.M., Boyce, M.C., 1993. A three-dimensional constitutive model for the large stretch behavior of rubber elastic materials. J. Mech. Phys. Solids 41, 389-412.

Atkins, G.J., Welldon, K.J., Holding, C.A., Haynes, D.R., Howie, D. W., Findlay, D.M., 2009. The induction of a catabolic phenotype in human primary osteoblasts and osteocytes by polyethylene particles. Biomaterials 30, 3672-3681.

Ayoub, G., Zaïri, F., Naït-Abdelaziz, M., Gloaguen, J.M., 2010. Modelling large deformation behaviour under loadingunloading of semicrystalline polymers: application to a high density polyethylene. Inter. J. Plasticity 26, 329-347.

Ayoub, G., Zaïri, F., Fréderix, C., Gloaguen, J.M., Naït-Abdelaziz, M. Seguela, R., Lefebvre, J.M., 2011. Effects of crystal content on the mechanical behaviour of polyethylene under finite strains: experiments and constitutive modelling. Int. J. Plasticity 27 , 492-511.

Ben Hadj Hamouda, H., Laiarinandrasana, L., Piques, R., 2007. Viscoplastic behaviour of a medium density polyethylene (MDPE): constitutive equations based on double nonlinear deformation model. Int. J. Plasticity 23, 1307-1327.

Bergström, J.S, Kurtz, S.M., Rimnac, C.M., Edidin, A.A., 2002. Constitutive modeling of ultra-high molecular weight polyethylene under large-deformation and cyclic loading conditions. Biomaterials 23, 2329-2343.

Boyce, M.C., Socrate, S., Llana, P.G., 2000. Constitutive model for the finite deformation stress-strain behavior of poly(ethylene terephthalate) above the glass transition. Polymer 41, 2183-2201.

Chaparro, B.M., Thuillier, S., Menezes, L.F., Manach, P.Y., Fernandes, J.V., 2008. Material parameters identification: gradient-based, genetic and hybrid optimization algorithms. Comput. Mater. Sci. 44, 339-346.

Drozdov, A.D., 2009. Mullins' effect in semicrystalline polymers. Int. J. Solids Struct. 46, 3336-3345.

Dusunceli, N., Colak, O.U., 2008. Modelling effects of degree of crystallinity on mechanical behavior of semicrystalline polymers. Int. J. Plasticity 24, 1224-1242.

Fouad, H., Elleithy, R., 2011. High density polyethylene/graphite nano-composites for total hip joint replacements: processing 
and in vitro characterization. J. Mech. Behav. Biomater. 4, 1376-1386.

Gen, M., Cheng, R., 2000. Genetic Algorithms and Engineering Optimization. John Wiley.

G'Sell, C., Dahoun, A., Royer, F.X., Philippe, M.J., 1999. The influence of the amorphous matrix on the plastic hardening at large strain of semicrystalline polymers. Model. Simul. Mater. Sci. Eng. 7, 817-828.

Hachour, K., Zaïri, F., Naït-Abdelaziz, M., Gloaguen, J.M., Aberkane, M., Lefebvre, J.M., 2014. Experiments and modeling of high-crystalline polyethylene yielding under different stress states. Inter. J. Plasticity 54, 1-18.

Mahmoudi, A.H., Pezeshki-Najafabadi, S.M., Badnava, H., 2011. Parameter determination of Chaboche kinematic hardening model using a multi objective Genetic Algorithm. Comput. Mater. Sci. 50, 1114-1122.

Mano, J.F., Sousa, R.A., Boesel, L.F., Neves, N.M., Reis, R.L., 2004. Bioinert, biodegradable and injectable polymeric matrix composites for hard tissue replacement: state of the art and recent developments. Compos. Sci. Technol. 64, 789-817.

Michalewicz, Z., 1999. Genetic Algorithms+Data Structures= Evolution Programs. Springer.

Miettinen, K., Neittaanmäki, P., Mäkelä, M.M., Périaux, J., 1999. Evolutionary Algorithms in Engineering and Computer Science. John Wiley.

Nikolov, S., Doghri, I., Pierard, O., Zealouk, L., Goldberg, A., 2002. Multi-scale constitutive modeling of the small deformations of semi-crystalline polymers. J. Mech. Phys. Solids 50, 2275-2302.

Ponçot, M., Addiego, F., Dahoun, A., 2013. True intrinsic mechanical behaviour of semi-crystalline and amorphous polymers: Influences of volume deformation and cavities shape. Int. J. Plasticity 40, 126-139.

Pyrz, M., Zaïri, F., 2007. Identification of viscoplastic parameters of phenomenological constitutive equations for polymers by deterministic and evolutionary approach. Model. Simul. Mater. Sci. Eng. 15, 85-103.

Ramakrishna, S., Mayer, J., Wintermantel, E., Leong, K.W., 2001. Biomedical applications of polymer-composite materials: a review. Compos. Sci. Technol. 61, 1189-1224.

Regrain, C., Laiarinandrasana, L., Toillon, S., Saï, K., 2009. Multimechanism models for semi-crystalline polymer: constitutive relations and finite element implementation. Int. J. Plasticity 25, 1253-1279.

Schultz, J.M., 1984. Microstructural aspects of failure in semicrystalline polymers. Polym. Eng. Sci. 24, 770-785.

Seguela, R., 2007. On the natural draw ratio of semi-crystalline polymers: review of the mechanical, physical and molecular aspects. Macromol. Mater. Eng. 292, 235-244.

Simis, K.S., Bistolfi, A., Bellare, A., Pruitt, L.A., 2006. The combined effects of crosslinking and high crystallinity on the microstructural and mechanical properties of ultra high molecular weight polyethylene. Biomaterials 27, 1688-1694.

Uchida, M., Tada, N., 2011. Sequential evaluation of continuous deformation field of semi-crystalline polymers during tensile deformation accompanied by neck propagation. Int. J. Plasticity 27, 2085-2102.

Zaïri, F., Naït-Abdelaziz, M., Woznica, K., Gloaguen, J.M., 2005. Constitutive equations for the viscoplastic-damage behaviour of a rubber-modified polymer. Eur. J. Mech. A/Solids 24, 169-182.

Zaïri, F., Aour, B., Gloaguen, J.M., Naït-Abdelaziz, M., Lefebvre, J. M., 2006. Numerical modelling of elastic-viscoplastic equal channel angular extrusion process of a polymer. Comput. Mater. Sci. 38, 202-216.

Zaïri, F., Naït-Abdelaziz, M., Gloaguen, J.M., Lefebvre, J.M., 2011. A physically-based constitutive model for anisotropic damage in rubber-toughened glassy polymers during finite deformation. Inter. J. Plasticity 27, 25-51. 